\title{
Stochastic Day-ahead Optimal Scheduling of Active Distribution Networks with Dispersed Energy Storage and Renewable Resources
}

\author{
M. Nick, Student Member, IEEE, R. Cherkaoui, Senior Member, IEEE, and M. Paolone, Senior Member, IEEE \\ Distributed Electrical System Laboratory (DESL) \\ École Polytechnique Fédérale de Lausanne EPFL, Lausanne, Switzerland \\ \{mostafa.nick; rachid.cherkaoui; mario.paolone\}@epfl.ch
}

\begin{abstract}
This paper focuses on the problem of the probabilistic optimal day-ahead scheduling of energy resources in Active Distribution Networks (ADNs). These resources include both dispersed energy storage systems (DESSs) and volatile renewable embedded generators. Technical constraints related to both energy resources and electrical network are modeled and taken into account in the proposed optimization problem. The paper first proposes a convex formulation of a specific optimal power flow (OPF) used to compute the resources schedule. Its objective function aims at achieving the minimum of the following quantities: network and DESSs losses, energy cost imported from the external grid, and deviations from the day-ahead scheduled power flow with the same external grid. In addition, the ability of using the substation transformer tap-changer is incorporated into the problem with a suitable cost function. The initial OPF formulation is then enhanced thanks to the use of the Mixed Integer Second Order Cone Programming approach in order to formulate a stochastic AC-OPF. The uncertainties of the problem are due to the forecast errors of the PV generation, load consumption and energy prices. The applicability and the effectiveness of the proposed scheduling approach are tested by using a modified version of the IEEE 34 buses test feeder.
\end{abstract}

Keywords - dispersed energy storage systems; active distribution networks; stochastic programming; convex optimization, optimal power flow.

\section{NOMENCLATURE}

A. Parameters
$E_{\text {max }}, E_{\text {min }}$
$P_{\text {max }}$
$v_{\text {max }}, v_{\text {min }}$
$f_{i j, \max }$
$z_{i j}$
$r_{i j}$

Maximum/Minimum BESSs State-ofCharge $(\mathrm{SoC})$ Maximum active power output of BESSs Maximum/Minimum limits of squared network nodal voltages Maximum limit of squared current flow rating between buses $i$, and $j$ Impedance of the line between buses $i$, and $j$ Longitudinal resistance of the line

$W_{\text {Tap }}, W_{E}, W_{L}, W_{L C}$
$\gamma$
$\rho_{S c}$
$C_{S}$
$r_{s}$
$\pi$
$s_{i}$

between buses $i$, and $j$

Weighting coefficient of the terms composing the objective function Penalty factor for day-ahead scheduling deviation

Probability of scenario $S c$

Maximum power rating of BESS $s$

Resistive loss factor of BESSs

Energy price from the external grid

Net apparent power injected/absorbed at bus $i$

\section{B. Variables}

TC

$T P$

$E_{S c}$

$E^{D A}$

$f_{i j}$

$L_{S}$

$S_{i j}$

$v_{i}$

$E_{S}$

$P_{S}, Q_{s}$

$L C_{i}$

$S_{i}{ }^{g e r}$

C. Indices

$t$

Sc

$i, h$
Cost associated to the changes of position of substation transformer tap-changer Substation transformer tap position

Energy imported from the external grid in scenario $S c$

Energy scheduled exchange with the external grid in day-ahead

Square of current flow over line $i j$

Resistive losses of BESS $s$

Apparent power flow over line $i j$

Square of nodal voltage at bus $i$ ( 0 is the index of slack bus)

Energy stored in BESS $s$

Active/Reactive power

production/consumption of BESS $s$

Load curtailment at bus $i$

Apparent power of BESS connected to bus $i$

Index of time steps

Index of scenarios

Index of buses 
$i j$

$S$

\section{Sets}

$G$

ESS

$N$

Index of line between buses $i$ and $j$

Index of BESSs

Set of the lines

Set of BESSs

Set of the network buses

\section{INTRODUCTION}

As discussed in the recent literature, the control philosophies of the so-called Active Distribution Networks (ADNs) are rapidly evolving as a consequence of the envisioned optimal control of the dispersed energy resources connected to this layer of the electrical infrastructure (e.g., [1], [2]). In this respect, one of the potential leverages is represented by the availability of Dispersed Energy Storage systems (DESSs). In general, these systems have the capability to provide ancillary services by controlling both active and reactive power in sub-second time intervals and on the four quadrants. Large Battery Energy Storage Systems (BESSs) are the nowadays most adopted DESSs.

Within the context of optimal operation of ADNs, one of the approaches that have been proposed in the literature refers to the optimal day-ahead scheduling of embedded energy resources (e.g., [3]). In this respect, the optimal day-ahead scheduling can be approached by solving specific Optimal Power Flow (OPF) problems. The category of OPF-problems can be formulated with different objective functions, like minimization of: voltage deviations, line congestions, network losses, or energy-cost. As known, OPF-problems are inherently non-convex and, when accounting for the possibilities to control resources with discrete states (e.g., transformer tap-changers), they become Mixed Integer Non Linear Problems (MINLP). As known, MINLP are hard to solve and computationally expensive where, additionally, the finding of the global optimal solution is not guaranteed. At the same time, the presence of volatile renewable resources introduces uncertainties due to the errors in their day-ahead production forecasts. This aspect makes the problem even more complex as it includes stochastic processes.

In this respect, it is worth mentioning that, since uncertainties may cause financial losses to the Distribution Network Operators (DNOs), they should be considered in the day-ahead scheduling. Indeed, DESSs, in addition to the network supports capabilities and energy arbitrage benefits, can be used to reduce the power flow deviations with the external grid with respect to the day-ahead schedule.

Several approaches have been used to solve the problem of day-ahead scheduling of ADNs. The Authors of [4] proposed a stochastic day-ahead scheduling that considers the uncertainties of the renewable energy resources. A two stage optimal scheduling procedure for distribution networks is proposed in [3] where the optimal set-points of the distributed resources are determined in a dedicated day-ahead scheduler where an intraday controller solves, each 15 minutes, another optimization problem aiming at satisfying specific operation objectives (i.e., voltage profiles, losses minimization etc.). A day-ahead resource scheduling in ADNs with high penetration of electrical vehicles and embedded generation is presented in [5] where the problem is solved by taking advantage of a heuristic technique based on the Particle Swarm Optimization (PSO). A specifically defined active-reactive power flow optimization is proposed in [6]. It aims at minimizing the total cost of the supplied electricity and the total network losses. The problem is formulated for a given ADNs assuming the presence of embedded generation and battery energy storage systems.

The above-mentioned papers either propose partially nonconvex formulation of the OPF problem (requiring the use of heuristic techniques) or address only the economic dispatch problem without considering the technical constraints of the network. In distribution networks characterized by a purely radial structure, some relaxations have been proposed in order to make the problem convex. A convex relaxation for OPF problem is proposed in [7] and it is proven to be exact with the over-satisfaction of supplied load. In [8] a sufficient condition is proposed for checking the exactness of the relaxed OPF problem solution. In this last paper, the Authors suggested a relaxation with more conservative constraints for the voltage. An exact convex relaxation of optimal power flow problem is proposed in [9] for radial distribution networks.

By using the method of [9], in this paper we propose a stochastic method to solve the day-ahead optimal scheduling problem of radial ADNs in presence of BESSs and volatile renewable resources. BESSs are modeled by representing their State-of-Charge $(\mathrm{SoC})$ and resistive losses, as well as their capabilities, to support the network by variable injected/absorbed active/reactive powers on the four quadrants. The stochastic daily optimal power flow scheduling is formulated as a stochastic Mixed Integer Quadratically Constrained Quadratic Program (MIQCQP). The possibility to control the substation transformer tap-changer is also accounted for.

The rest of the paper is structured as follows: the complete formulation of the problem is described in section II. An exhaustive analysis of the algorithm performances is presented in Section III with reference to a modified IEEE 34 bus test feeder. A discussion concerning DNOs benefits associated to the use of the proposed method concludes the paper.

\section{PROBLEM DESCRIPTION}

As anticipated above, the exact convex relaxation of OPF problem for radial distribution networks proposed in [9] is used in this paper. The lines are modeled using the classical two-port $\Pi$ equivalents. Thus, the reactive power associated to shunt impedance of the lines is also considered.

Concerning the BESSs, since they are normally interfaced to the grid by means of power electronic converters, we have assumed their ability to support the network by controlling both active and reactive powers. BESS capability curves are included in the model by a cone constraint. The losses taking place into the BESSs converter are also considered. They are considered by a relaxed second order cone constraint. 


\section{A. Uncertainties of the model}

The sources of uncertainties are associated to the forecasted photovoltaic (PV) production, load and price profiles from the day-ahead market. To deal with these uncertainties, various scenarios are considered for load, price and PV productions. A stochastic scenario tree is built based on these inputs. Then, a two stage stochastic programming approach is used to find the optimal scheduling $[10,11]$. The decision of the first stage is made when the random variables are unknown (in the dayahead). Then, the second stage decision (in real time) is performed. The random variables of this second stage are the PV production, load consumption and energy price. It is worth noting that an inherent coupling between the two stages exists since this last one is influenced by the decisions made in the former stage.

We assume that the first-stage decision declares the amount of import/export energy from the external grid at each hour in the day-ahead market. The operation and control of the grid for various scenarios compose the second stage where the decision variables are the amount of active and reactive power that BESSs produce at each time step.

\section{B. Mathematical formulation of the problem}

The objective function of the problem (as it is shown in (1)) accounts for the minimization of different aims: (i) energy cost from the external grid, (ii) penalty deviations from the dayahead schedule of the energy import/export from/to external grid, (iii) cost of changing the transformer tap-changer position and (iv) total network and BESSs losses. It is worth noting that objectives (i) and (ii) might compete. However, the inclusion of both of these two objectives allow the DNOs to enable conditions for which the economical losses associated to large deviations from the day-ahead schedule are largely compensated by the exported power towards the external grid.

The voltage deviations are incorporated into the model by a set of constraints that limit its variation to $5 \%$. The mathematical formulation of the optimization problem is as follow:

$$
\begin{aligned}
\min \rho_{S c} \sum_{S c} \sum_{t}\{ & W_{T a p} T C_{S c}(t)+W_{E}\left(E_{S c}(t) \pi_{S c}(t)\right. \\
& \left.+\gamma_{S c, t}\left|E^{D A}(t)-E_{S c}(t)\right|\right) \\
& +W_{L}\left[\left(\sum_{i j \in G} r_{i j} f_{i j, S c}(t)\right)+\sum_{s} L_{s, S c}(t)\right] \\
& +W_{L C} \sum_{i}^{\left.L C_{i, S c}\right\}}
\end{aligned}
$$

Subject to:

$$
\begin{array}{r}
S_{i j, S c}(t)=s_{i, S c}(t) \\
+\sum_{\substack{h: h \rightarrow i \\
\text { ger }}}\left(S_{h i, S c}(t)-z_{h i} f_{h i, S c}(t)\right) \\
-S_{i, S c}(t)-L C_{i, S c} \forall(i, j) \in G
\end{array}
$$

$$
\begin{aligned}
& v_{i, S c}(t)=v_{j, S c}(t)+2 \operatorname{Re}\left(\bar{z}_{i j} S_{i j, S c}(t)\right)-\left|z_{i j}\right|^{2} f_{i j, S c}(t) \\
& \forall(i, j) \in G \\
& 0=s_{0, S c}+\sum_{h: h \rightarrow 0}\left(S_{h 0, S c}-z_{h 0} f_{h 0, S c}\right) \\
& f_{i j, S c}(t) \geq \frac{\left|S_{i j, S c}(t)\right|^{2}}{v_{i, S c}(t)} \quad \forall(i, j) \in G \\
& f_{i j, S c}(t) \leq f_{i j, \max } \quad \forall(i, j) \in G \\
& v_{\text {min }} \leq v_{i, S c}(t) \leq v_{\text {max }} \quad i \in N \\
& E_{S, S c}(t+1)=E_{s, S c}(t)-P_{s, S c}(t)-L_{s, S c}(t) \forall s \\
& \in E S S \\
& -P_{s, \max } \leq P_{S, S c}(t) \leq P_{S, \max } \quad \forall s \in E S S \\
& E_{s, \text { min }} \leq E_{S, S c}(t) \leq E_{S, \max } \quad \forall s \in E S S \\
& P_{s, S c}^{2}(t)+Q_{S, S c}^{2}(t) \leq C_{S}^{2} \quad \forall s \in E S S \\
& L_{s, S c}(t) \geq r_{s}\left(P_{s, S c}^{2}(t)+Q_{s, S c}^{2}(t)\right) \quad \forall s \in E S S \\
& v_{0}=1+0.01\left(T P_{S c}(t)\right) \\
& 0 \leq T P_{S c}(t) \leq 10 \\
& T P_{S c}(t): \text { integer } \\
& T C_{S c}(t) \geq 0 \\
& T C_{S c}(t) \geq T P_{S c}(t)-T P_{S c}(t-1) \\
& T C_{S c}(t) \geq-T P_{S c}(t)+T P_{S c}(t-1)
\end{aligned}
$$

The equations (2), (3), (4), (5) and (6) are related to the load balance and flow limits in the network feeders. In [9] it is proven that if two specific conditions hold, then the solution of the relaxed OPF problem is exact. For sake of brevity, we briefly recall the first condition ${ }^{1}$. It states that the upper limit of the voltage should not be limiting. In this respect (4) is added to the problem to ensure that the upper limit of the voltage is not binding. This slightly shrinks the solution space of the problem ${ }^{2}$. The constraint (7) models the upper and lower limits of the bus voltages. The $S o C$ of the BESSs is modeled as shown in (8) and their power rating and reservoir capacity limits are modeled by (9) and (10) ${ }^{3}$.

The capability curve of the BESSs is modeled by (11). It should be noted that it is assumed that the reactive power regulation done by the BESSs does not affect their SoC however, it impacts the BESS resistive losses. Equation (12) represents the relaxed version of the BESSs resistive losses, which is originally an equality constraint instead of an inequality one. This relaxation is exact since these losses are

\footnotetext{
${ }^{1}$ The second condition can be checked a priori. It has been proven to hold for IEEE 34 buses test system with high penetration of DGs (actually it holds for most of the radial distribution networks even with high penetration of renewable energy.) [9].

${ }^{2}$ For further details, see [9].

${ }^{3}$ It is worth noting that the optimal siting and sizing of DESS has been
} discussed in [12]. 
minimized in the objective function.

The tap-change of the sub-station transformer is modeled by the equations (13) - (18). It is assumed it has 5 steps for regulation such that each one can regulate the voltage of $1 \%$. In the next section the proposed model is examined with standard test case study and various scenarios. In the following section we compare the behavior of the network with and without BESSs. The later case is analyzed by simply forcing to zero the energy/power capacities of BESSs in equations (9-11).

\section{SimULATION RESUlTS}

As it is shown in Fig. 1, in this section we have adopted a case study based on a modified IEEE 34 test distribution feeder. We compared two cases, i) no BESS is installed in the network, ii) two BESSs are optimally located in the network using the procedure described in [4]. It is assumed that the PV panels are installed on the roof of the customers therefore it is distributed among the buses based on the their loading. The percentages of load and PV connected to bus " $i$ ", with respect to the total one, is shown in Table I.

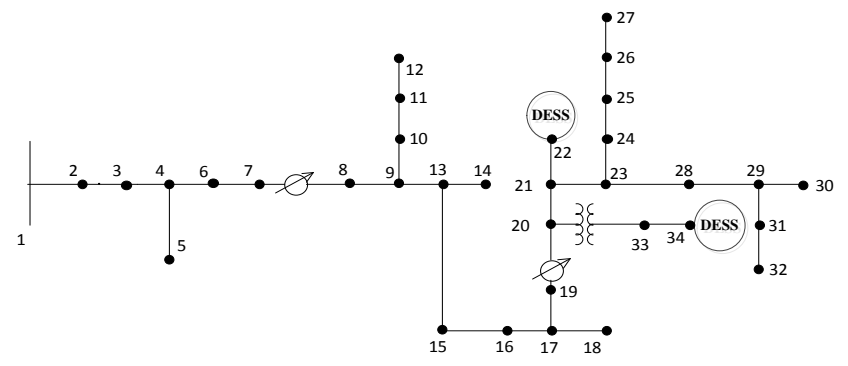

Fig. 1: Schematic of the modified IEEE 34 buses test system.

TABLE I. LOAD/PV SHARE OF EACH BUS

\begin{tabular}{cccccc}
\hline $\begin{array}{c}\text { Node } \\
\#\end{array}$ & $\begin{array}{c}\text { Percentages } \\
\text { of total } \\
\text { load/PV }\end{array}$ & Bus \# & $\begin{array}{c}\text { Percentages } \\
\text { of total } \\
\text { load/PV }\end{array}$ & Bus \# & $\begin{array}{c}\text { Percentages } \\
\text { of total } \\
\text { load/PV }\end{array}$ \\
\hline 3 & 6.5 & 5 & 0.9 & 11 & 1.92 \\
\hline 12 & 7.63 & 13 & 0.28 & 14 & 2.26 \\
\hline 15 & 0.23 & 16 & 2.94 & 18 & 0.22 \\
\hline 21 & 0.85 & 22 & 0.11 & 23 & 1.80 \\
\hline 25 & 23.40 & 26 & 2.54 & 27 & 4.69 \\
\hline 28 & 8.25 & 29 & 4.64 & 30 & 3.79 \\
\hline 32 & 1.58 & & & 34 & 25.44
\end{tabular}

A total of 20 scenarios are assumed to cover the uncertainties of the PV production using real measurements. Similarly, 10 scenarios are provided for daily load variations. These scenarios are built using Monte-Carlo simulation with 5\% standard deviation. These two sets provide 200 scenarios in total for the aggregated net load of the system (PV production and load). The K-Means method [13] is used to decrease these 200 scenarios to 30. As known, the method tries to synthesize the $k$ clusters so that the mean squared distance from each data of the original set and the synthesized clusters is minimal. The clustered scenarios related to the total net load of the network are shown in Fig. 2. The uncertainties of the price variations are also considered with 10 scenarios that are shown in Fig. 3. The reduced scenarios of total net load (30 ones) and the price scenarios define the scenario tree of the optimization problem. It produces 300 scenarios in total. The weighing coefficients $(W)$ of each term of the objective function are calculated using Analytical Hierarchy Process (AHP) [14]. It should be noted that the weight of the load curtailment is considered to be a big number and it is not incorporated into the AHP. The simulation parameters are shown in Table II. The base value for power/energy is $2.5 \mathrm{MW} / \mathrm{MWh}$.

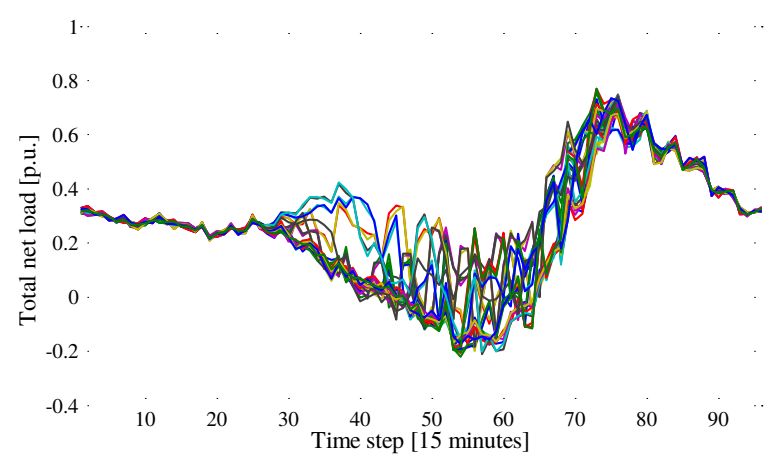

Fig. 2: Total net daily active load scenarios (aggregated of the load and PV generation). The base power is $2.5 \mathrm{MW}$.

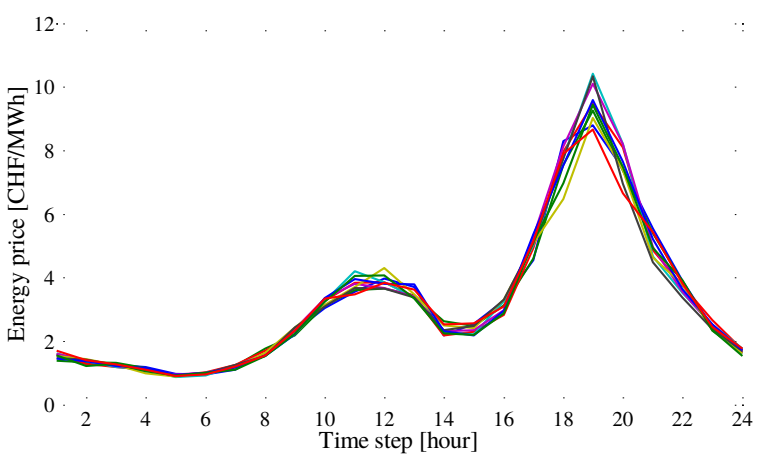

Fig. 3: Energy price scenarios.

TABLE II. SIMULATION PARAMETERS

\begin{tabular}{cccc}
\hline $\begin{array}{c}\text { BESS 1 power rating } \\
\text { [p.u.] }\end{array}$ & 0.15 & BESS 2 power rating [p.u.] & 0.17 \\
\hline $\begin{array}{c}\text { BESS 1 reservoir } \\
\text { capacity [p.u.] }\end{array}$ & 0.3 & $\begin{array}{c}\text { BESS 2 reservoir capacity } \\
\text { [p.u.] }\end{array}$ & 0.5 \\
\hline$W_{\text {Tap }}$ & 0.603 & $W_{E}$ & 0.23 \\
\hline$W_{L}$ & 0.167 & $W_{L C}$ & 100
\end{tabular}

The proposed MIQCQP model is implemented in YALMIPMATLAB interface [15] and is solved using Gurobi optimization software [16].

The objective function terms are reported in Table III for both cases. It shows that the total losses and the energy cost from the external grid are decreased using BESSs. In addition to these quantities, the total upward deviation (consuming/producing less/more energy than day-ahead schedule) and downward deviation (consuming/producing 
TABLE III. COMPARISON OF EACH TERM OF THE OBJECTIVE FUNCTION (BASE VALUE FOR POWER/ENERGY ARE 2.5 MW/MWH)

\begin{tabular}{|c|c|c|c|c|c|c|}
\hline & $\begin{array}{l}\text { Total average } \\
\text { loss in whole } \\
\text { scenarios [p.u.] }\end{array}$ & $\begin{array}{l}\text { Use of Tap- } \\
\text { changer }\end{array}$ & $\begin{array}{l}\text { Load curtailment } \\
\text { [p.u.] }\end{array}$ & $\begin{array}{l}\text { Total Upward } \\
\text { deviation [p.u.] }\end{array}$ & $\begin{array}{c}\text { Total Downward } \\
\text { deviation [p.u.] }\end{array}$ & $\begin{array}{l}\text { Energy cost } \\
\text { from the } \\
\text { external grid } \\
{[\mathrm{CHF}]}\end{array}$ \\
\hline With BESSs & 0.2138 & No & 0 & 2 & 1.23 & 201.22 \\
\hline $\begin{array}{l}\text { Without } \\
\text { BESSs }\end{array}$ & 0.29 .9 & YES & 0.85 & 3.17 & 1.8 & 246.34 \\
\hline
\end{tabular}

more/less energy than day-ahead schedule) are decreased significantly (these values are the total deviations for the all 30 load scenarios.). The Table III also shows that by using BESSs the sub-station transformer tap-changer is not used. On the contrary, it is used largely to modify the slack bus voltage for the case $\mathrm{i}$ (without the BESSs) For the case without the BESSs, $2.125 \mathrm{MWh}$ energy is to be curtailed in average in whole scenarios. The load curtailment is prevented with the presence of BESSs.

The voltage deviation is not directly included in the objective function and the maximum voltage deviation is limited to $5 \%$ by constraints (7). The boxplot of nodal voltages for each time step for both cases are shown in Fig. 4. The bottom and top of the blue boxes indicate the first and third quartiles $\left(25^{\text {th }}\right.$ and $75^{\text {th }}$ percentiles of the data) of the nodal voltages at each time step. The red lines indicate the median while the whiskers show the maximum and minimum of nodal voltages at each time step. It can be observed that, for the case without BESSs the $\mathrm{max} / \mathrm{min}$ of voltages are equal to 1.025 and
0.95 p.u. respectively. The max voltage corresponds to the value set at the slack bus by the tap-changer. The min voltage corresponds to the minimum limit associated to the constraint (7). This figure shows that the voltage profile of the network has been improved (i.e., the voltage deviations are minimized) by using BESSs.

The daily active power scheduling of the BESSs and their maximum/minimum variations in different scenarios are shown in Fig. 5. These variations are the capacities that are considered as the reserve for compensating the day-ahead forecast errors. As it can be seen from this figure, the reserve capacity in correspondence of the middle of the day is high in order to compensate the PV production forecast errors (the uncertainties of the PV production is much higher that the load ones).

\section{CONCLUSION}

The paper has proposed a process to solve the problem of the probabilistic day-ahead scheduling of energy resources connected to ADNs. The considered resources are DESSs (with
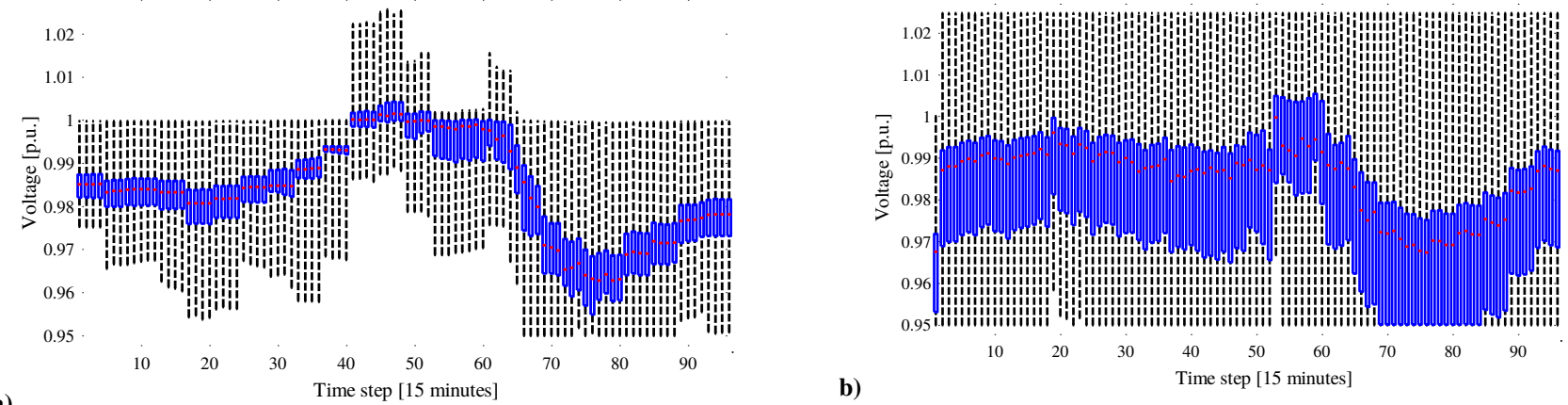

Fig. 4: The boxplot of the IEEE 34 test feeder nodal voltages. a) With BESSs and b) without BESSs.
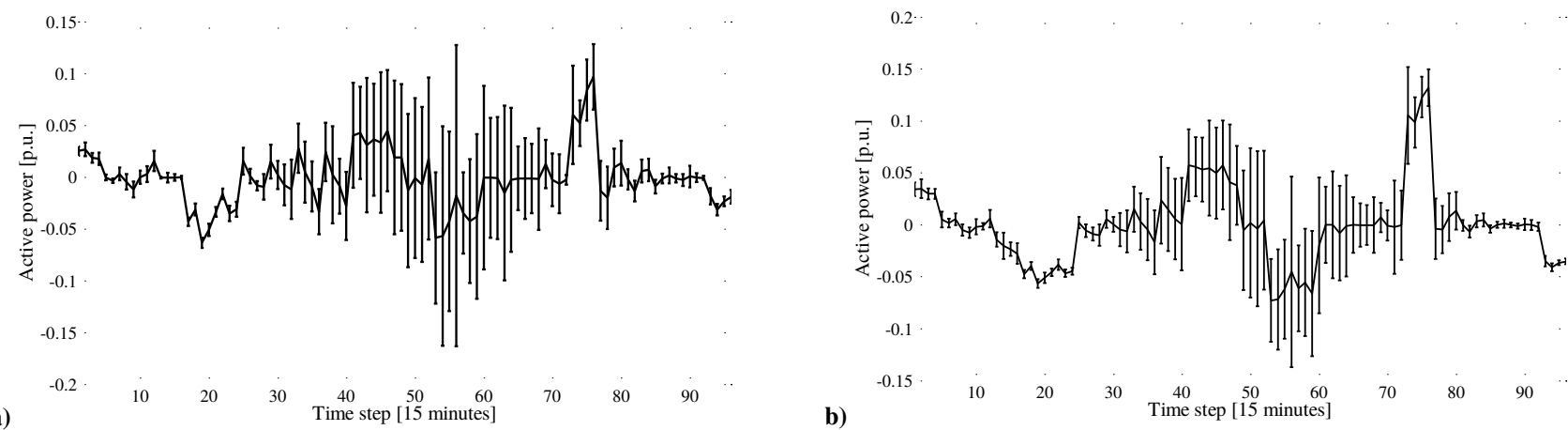

Fig. 5: BESSs daily active power schedule together with their maximum and minimum variations (base power equal to $2.5 \mathrm{MW}$ ). a) BESS 1 , b) BESS 2 
particular reference to BESSs) and non-dispatchable DGs. The technical constraints of the resources, together with the ones of the electrical network, are modeled and incorporated into the optimization problem using a method already presented in the literature. The objective function includes loss minimization and economical goals associated to the energy cost imported from external grid and the operation costs of the BESSs together with transformer tap-change cost function and deviation penalties due to deviation from day-ahead scheduling of the power flow with the external grid.

The uncertainties of load and PV forecast, in addition to the price forecast errors, are used to generate scenarios and formulate a stochastic MISOCP problem. The results show that the optimally controlled BESSs not only improve the quality of the service (i.e., decrease of voltage deviation) but also reduce the total losses and the day-ahead scheduling mismatch with respect to real-time scenarios. In addition, the proposed optimal control of these resources enables the load shifting and decreases the cost of energy supplied by the external subtransmission network.

\section{REFERENCES}

[1] N. Jenkins, R. Allan, P. Crossley, D. Kirschen, and G. Stcbac, Embedded Generation. London, U.K.: Inst. Elect. Eng., 2000.

[2] J. A. P. Lopes, N. Hatziargyriou, J. Mutalc, P. Djapic, and N. Jenkins, "Integrating distributed generation into electric power systems: A review of drivers, challenges and opportunities," Elect. Power Syst. Res., vol. 77, pp. 1189-1203, Jul. 2007.

[3] A. Borghetti, M. Bosetti, S. Grillo, S. Massucco, C. A. Nucci, M. Paolone, F. Silvestro, "Short-Term Scheduling and Control of Active Distribution Systems With High Penetration of Renewable Resources" IEEE Syst. Journal, vol. 4, pp. 313-322, Sep. 2010

[4] C. Jin, M. N. Mojdehi, P. Ghosh "A Methodology to Design a Stochastic Cost Efficient DER Scheduling Considering Environmental Impact"
International conference on Smart Grid, Syracuse, NY, USA, 27-29 Aug. 2012 pp. 1-6.

[5] J. Soares, H. Morais, T. Sousa, Z. Vale, P. Faria, "Day-Ahead Resource Scheduling Including Demand Response for Electric Vehicles" IEEE Trans. Smart grid, vol. 4, pp. 596-605, Mar. 2013.

[6] A. Gabash, P. Li, "Active-Reactive Optimal Power Flow in Distribution Networks With Embedded Generation and Battery Storage" IEEE Trans. Power Syst., vol. 27, pp. 2026-2035, NOV. 2012.

[7] M. Farivar, C. R. Clarkey, S. H. Low, K. M. Chandy, "Inverter VAR Control for Distribution Systems with Renewables" International conference on smart grid communication, Brussels, Belgium, 17-20 Oct. 2011, pp. $457-462$.

[8] L. Gan, N. Li, U. Topcu, S. Low "On the Exactness of Convex Relaxation for Optimal Power Flow in Tree Networks" IEEE Annual conference o decision and control, Grand HI, USA, 10-13 Dec 2012, pp. $465-471$

[9] L. Gan, N. Li, U. Topcu, and S. H. Low, "Exact Convex Relaxation of Optimal Power Flow in Radial Networks" arXiv preprint arXiv, 2012.

[10] J. G. González, R. M. Muela, L. M. Santos, and A. M. González, "Stochastic Joint Optimization of Wind Generation and Pumped-Storage Units in an Electricity Market," IEEE Trans. Power Syst., vol. 23, pp. 460-468, MAY 2008.

[11] I. G. Moghaddam, M. Nick, F. Fallahi, M. Sanei, and S. Mortazavi, "Risk-averse profit-based optimal operation strategy of a combined wind farm-cascade hydro system in an electricity market," Renewable Energy, vol. 55, pp. 252-259, Jul. 2013.

[12] M. Nick, R. Cherkaoui, and M. Paolone, "Optimal Allocation of Dispersed Energy Storage Systems in Active Distribution Networks for Energy Balance and Grid Support," IEEE Trans. Power Syst., in press.

[13] A. K. Jain, "Data clustering: 50 years beyond K-means," Pattern Recognition Letters, vol. 31, pp. 651-666, Jan. 2010.

[14] Thomas L. Saaty, "Decision making - the Analytic Hierarchy and Network Processes (AHP/ANP)," Journal of Systems Science and Systems Engineering, vol. 13, pp. 1-35, Mar. 2004.

[15] J. Löfberg, "YALMIP: A Toolbox for Modeling and Optimization in MATLAB," In Proceedings of the CACSD Conference, Taipei, Taiwan, 2004.

[16] Gurobi Optimization, Inc. "Gurobi Optimizer Reference Manual” 2012, available at: http://www.gurobi.com 